\title{
Vegetarian Diet - Dealing with Efficiency and Deficiency of It - A Review
}

\author{
Lata Kanyal Butola ${ }^{1}$, Deepika Kanyal², Ranjit Ambad³, \\ 1Department of Biochemistry, Jawaharlal Nehru Medical College, DMIMS, Sawangi, Meghe, \\ Wardha, Maharashtra, India, ${ }^{2}$ Department of Hospital Administration, Jawaharlal Nehru Medical \\ College, DMIMS, Sawangi, Meghe, Wardha, Maharashtra, India. ${ }^{3}$ Department of Biochemistry, \\ Datta Meghe Medical Sciences, Wanadongri, Nagpur, Maharashtra, India.
}

\section{ABSTRACT}

\section{BACKGROUND}

There is already a large amount of evidence demonstrating the health benefits of vegetarian and plant-based diets along with lower incidence of obesity, diabetes, heart disease and certain forms of cancer as well as improved lifespan. Vegetarian diets tend to be lower in fat, particularly saturated fat and higher in dietary fiber. Consuming more whole grains, legumes, nuts, soy protein along with the absence of red meat, this form of eating plan will have many benefits for the prevention and treatment of obesity and chronic health conditions, including diabetes and cardiovascular disease (CVD). Whereas an excellently-planned vegetarian or vegan diet may fulfil all nutritional needs of an individual person. It may be important to pay careful attention to certain nutrients to maintain an appropriate intake, especially if the person is on a vegan diet. Legumes, such as soy, dried beans, peas, and lentils, are also important for a vegan diet because they are great sources of sugar, protein, iron, zinc, and calcium. It is important to ingest nuts and seeds as they increase essential fat intake and help to meet the energy and protein needs. Fortified foods (including vitamin B-12, calcium, and vitamin D) must be eaten since they are the only acceptable sources for certain vitamins in the vegan diet. This article will review the evidence of the health benefits of a vegetarian diet and also address strategies for meeting the nutritional needs of those adopting a vegetarian or plantbased eating pattern, and summarize the characteristics and benefits of vegetarian diets in the general population and the possible beneficial effects of such diet on phosphate balance, insulin sensitivity, and the control of metabolic acidosis in chronic kidney disease (CKD) patients.

\section{KEY WORDS}

Nutrients, Vitamin B12, Zinc, Omega 3 Fatty Acids
Corresponding Author: Dr. Lata Kanyal Butola Department of Biochemistry, Jawaharlal Nehru Medical College, DMIMS, Karnataka, Sawangi, Meghe, Wardha, Maharashtra, India. E-mail: kanyallata1010@gmail.com

DOI: $10.14260 / \mathrm{jemds} / 2021 / 728$

How to Cite This Article:

Butola LK, Kanyal D, Ambad R. Vegetarian diet - dealing with efficiency and deficiency of it - a review. J Evolution Med Dent Sci 2021;10(41):3592-3597, $10.14260 /$ jemds/2021/728

Submission 20-10-2020, Peer Review 05-09-2021, Acceptance 13-09-2021, Published 11-10-2021.

Copyright (C) 2021 Lata Kanyal Butola et al. This is an open access article distributed under Creative Commons Attribution License [Attribution 4.0 International (CC BY 4.0)] 


\section{BACKGROUND}

What we eat greatly affects our personal health and the environment that we all share. Vegetarian diets are centred on foods derived from plants such as cereals, legumes, root crops, oilseeds, berries, potatoes, nuts, and mushrooms, as well as dairy and egg products. The various types of vegetarian diets planned share the same trend of avoiding meat, fish, and poultry consumption, while some also avoid eggs, milk, and dairy products. ${ }^{1}$ Lacto-ovo-vegetarians do not consume meat, poultry, or fish, but include dairy products and eggs on their diet; vegans do not consume animal-based food. In general, a vegetarian diet is lower in saturated fat than a non-vegetarian diet, and higher in starch, non-starch polysaccharide (NSP), fruits, and vegetables. ${ }^{2}$ Furthermore, the fact is that such foods include health-friendly ingredients such as fiber, phytoestrogens, antioxidants, phytochemicals, omega- 3 fatty acids and a limited amount of cholesterol has been well addressed; the ultimate impact is to minimize the risk of developing the following conditions; cardiovascular disease, cancer, diabetes and obesity. ${ }^{3}$

Several researches have pointed to the possibility of raising the human life span. ${ }^{4}$ If a vegetarian diet is insufficient, it is possible that dietary errors may occur during its adoption. $^{3}$ For a variety of factors, increased consumption of vegetables and fruits was accepted as policy of public health. Displacement of saturated fat and increased fiber intake is seen as general explanation for rising consumption of fruits and vegetables. Fruit and vegetables in the highly active "Dietary Approach to Stop Hypertension (DASH) program", combined with increased calcium intake and salt restriction in a diet, low in total fat and very low in saturated fat, showed significant results close to those with initial dose of single drug therapy in lowering blood pressure. Vegetables and fruits mainly decrease the loss of urinary calcium as sources of alkali. Vegetable replacement for animal protein can preserve kidney function. Fruits and vegetables gained more interest in CVD, as plant-based diet helps in minimizing the risk of loss from CVD. 5

\begin{tabular}{|c|c|}
\hline $\begin{array}{c}\text { Type of } \\
\text { Vegetarian } \\
\text { Diet }\end{array}$ & Definition \\
\hline $\begin{array}{l}\text { Lacto-ovo- } \\
\text { vegetarian }\end{array}$ & $\begin{array}{l}\text { Excludes all types of flesh foodstuffs (meat, poultry, fish), but } \\
\text { permits eating all other animal products (e.g. eggs, milk, honey). }\end{array}$ \\
\hline Lacto-vegetarian & $\begin{array}{c}\text { Excludes flesh foodstuffs and eggs but allows dairy products, } \\
\text { honey, etc. }\end{array}$ \\
\hline Ovo- vegetarian & $\begin{array}{c}\text { Excludes consumption of all animal products with the exception of } \\
\text { eggs. }\end{array}$ \\
\hline Vegan & Excludes all animal products. \\
\hline Pesco vegetarian & $\begin{array}{l}\text { Excludes meat and poultry, but includes fish (possibly other sea } \\
\text { food) }\end{array}$ \\
\hline Vitarian & $\begin{array}{l}\text { Permits consumption of organic, raw and fresh foods only. } \\
\text { Excludes coffee and tea. }\end{array}$ \\
\hline Liquidarian & Consumption of vegetarian food in the form of juices. \\
\hline Fruitarian & Excludes flesh foodstuffs, animal product and vegetables. \\
\hline Sproutarian & $\begin{array}{c}\text { Eating foods in the form of sprouted plant seedlings, such as grains, } \\
\text { vegetables, fruits. }\end{array}$ \\
\hline Semi-vegetarian & $\begin{array}{l}\text { Transitional form between vegetarian and meat-based diets; } \\
\text { limited amount of meat eaten. }\end{array}$ \\
\hline
\end{tabular}

The vegetarian diet is mainly associated with low levels of low-density lipoprotein (LDL) cholesterol. Many researchers focused on vegan diet including berries, leafy vegetables, nuts etc. and they have seen a significant reduction in LDL cholesterol by 25 - $30 \%$. The risk of diabetes and cardiovascular diseases is decreased by consuming cereal fiber and total consumption of fibers is associated with decreased risk factors for coronary artery diseases (CAD) and intake of whole grains tends to not only minimize the incidence of CAD and diabetes but also to minimize blood glucose, fasting insulin and evidence of insulin resistance in obese, insulin-resistant subjects. ${ }^{6}$

In addition, the bioavailability of phosphates from plant proteins is reduced. Compared to an omnivorous diet, a vegetarian diet is rich in fiber, magnesium, fe $3+$, folic acid, vitamins $\mathrm{C}$ and $\mathrm{E}$, omega- 6 polyunsaturated fatty acids, phytochemicals, and antioxidants but low in cholesterol; total fat and saturated fatty acids; sodium; Fe 2 +; zinc, vitamins A, B12 and D; and particularly omega-3 polyunsaturated fatty acids. $^{7}$

\section{Vegetarian Diet: What are the Benefits?}

\section{May Improve Mood}

Many placebo trial intervention studies have reported mood changes after vegetarian diets are adopted. Mechanisms that lead to this change were not investigated. The mood is regulated by the action of the serotonin, norepinephrine neurotransmitters, proinflammatory cytokines (e.g., IL-1 $\beta$, TNF- $\alpha$, IL-6, and IL-2) and eicosanoids (e.g., PGE2). Vegetarian diets are naturally rich in antioxidants, and adherence to vegetarian diet is associated with low concentration of oxidative stress and PGE2. In addition, the high ratio of vegetarian diets with carbohydrates to protein will promote tryptophan entry into the brain and serotonin production. ${ }^{8}$

\section{May Improve Symptoms of Psoriasis}

Psoriasis is an inflammatory skin disease mediated by $\mathrm{T}$ cells and is characterized by hyperproliferation and decreased epidermal keratinocyte differentiation. In many cases, it can result from inadequate nutritional status and may also be induced by interactions between drugs and nutrients and plays a role in pathogenesis and aetiology of psoriasis. ${ }^{9}$ Both vegetarian and vegan diets have been tested for their efficacy in relieving symptoms of chronic inflammatory disorders. While the 2 diets are similar in preventing meat, fish and poultry consumption, vegan diets also have additional food restrictions including the avoidance of eggs, honey and dairy products. It was found that certain patients with psoriasis reported an improvement in their symptoms during the time of consuming a vegetarian or vegan diet due to a return to normal neutrophil function, extrapolated from serum lactoferrin levels. ${ }^{10}$ Many patients with psoriasis are prone to gluten, and they are advised to take a gluten free diet. The active form of vitamin D has both immunoregulatory and antiproliferative effects and has shown positive effects in treatment of psoriasis. ${ }^{9}$

Vegetarian diets have been shown to be associated with higher anti-inflammatory ratios to proinflammatory adipokines relative to omnivorous diets ${ }^{11}$ as well as lower levels of pro-inflammatory genes in the gut microbiota and lower levels of IgE expression. ${ }^{12}$ The symptoms of psoriasis may have been affected by the anti-inflammatory effects of diets. The advantages of a vegetarian diet have also been linked to the high intake of potassium ${ }^{13}$ that is used in the body to synthesize cortisol, a common psoriasis medication. Supplementation with potassium has been shown to increase serum cortisol in patients. ${ }^{14}$

While further studies are required in order to determine the importance of potassium in the vegetarian diet, both 
theories are plausible explanations for the findings found in these trials.

\section{May Reduce Incidence of Diabetes}

World Health Organization (WHO) has predicted that by 2030 , Type 2 diabetes mellitus may affect up to 79.4 million individuals in India. ${ }^{15} \mathrm{~A}$ whole-food, eating style based on plants typically involves legumes, whole grains, fruits, vegetables, and nuts, which are high in fiber. All of those components were found to be diabetes-protective. Whole grains, including whole-grain bread, cereals, and brown rice, have been shown to reduce the risk of diabetes development. Unique fruits and vegetables have been related to lower diabetes rate including root vegetables, green leafy vegetables, blueberries, grapes, and apples. Legumes have also been shown to enhance insulin resistance and protect against metabolic syndrome, and increased use of nut has been associated with lower risk of diabetes. Cereal fiber appears to be especially protective against type 2 diabetes. ${ }^{16}$

\section{Reduces Risk of Cardiovascular Disease:}

One of the most leading cause of death world-wide is cardiovascular disease, accounting for 46 percent of deaths from non-communicable diseases. Vegetarian dietary habits minimize CVD deaths and coronary heart disease (CHD) risk by $40 \%$. Obesity has a major effect on cardiovascular disease. In many studies, the significantly reduced mean body mass index (BMI) reported in vegans may be an important protective factor for lowering blood lipids and lowering the risk of heart disease. Hence, a diet rich in fruit and vegetable intake, like the vegan diet, is believed to minimize concentrations of blood cholesterol, decrease the incidence and mortality rate of strokes, and decrease the risk of mortality from ischemic heart disease. Plant-based diets are associated with reduced blood pressure, lower blood lipids and lower platelet aggregation than non-vegetarian diets and are effective in weight control, lower risk of developing metabolic syndrome and type 2 diabetes. These have also been shown to be an important way of treating diabetes. Wellplanned vegetarian diets have benefits in preventing and reversing atherosclerosis and in reducing risk factors for CVD and should be encouraged by dietary recommendations and guidance. ${ }^{17,18}$ The main risk factor for cardiovascular disease, peripheral artery disease and stroke is dyslipidaemia. In patients with elevated concentrations of plasma cholesterol, existing recommendations prescribe diet as first-line therapy. Large prospective trials have shown that populations adopting plant-based diets, especially vegetarian and vegan diets, are at lower risk for mortality from ischemic heart disease. Study conducted by Hope R. Ferdowsian et al. evaluates the efficacy of plant-based diets in altering concentrations of plasma lipids. Which included 27 randomized controlled and observational trials. Among the 4 forms of plant-based diets examined, intervention testing of a hybrid diet (a vegetarian or vegan diet paired with nuts, soy and/or fiber) showed the greatest benefits (up to 35 percent reduction in plasma low-density lipoprotein cholesterol), accompanied by vegan and ovolactovegetarian diets. Strategies requiring small quantities of lean meat showed less drastic reductions in total cholesterol and low-density lipoprotein levels and found that plant-based dietary strategies are successful in lowering concentrations of plasma cholesterol. ${ }^{19}$

\section{Vegetarian Diet and Cancer}

Diet is one of the main causes of premature death and disability in developing countries and adds to the burden of cancers typically found in Western culture. Due to the strong dietary impact on cancer incidence and progression as well as the significant financial burden imposed by existing treatment schemes, avoidance by adherence to a primarily plant-based diet is an appealing way of tackling the problem. ${ }^{20}$ The claim is substantiated by a more rigorous review of the vegan diet for nutrients that are cancer protective foods. Vegans as compared to omnivores eat more legumes, onions, variety of fruits and vegetables, seeds, starch and citrus fruits, all of which are cancer protective.

Fruits and vegetables also protect against cancer of mouth, lung, stomach and oesophagus. Legumes are considered more effective against cancer of stomach and prostate. Phytochemicals present in fruits and vegetables have strong anti-oxidant and antiproliferative capacity and exhibits additive and synergistic associations with cancer progression involving cellular processes. In other words, the phytochemicals hinder cell proliferation, the formation of deoxy ribo nucleic acid (DNA) adducts, signal transduction pathways, oncogene expression, and cause cell cycle arrest and apoptosis.

\section{Nutrients You May Be Missing If You are Vegetarian}

\section{Vitamin $B 12$}

Vitamin B12 is also known as cobalamin, extrinsic factor of castle and anti-pernicious anaemia factor. Individuals adopting a plant-based diet that does not contain any animal products are vulnerable to B12 deficiency and need to supplement their diet with vitamin B12 or vitamin B12 fortified foods. Vitamin B12 is synthesized in gastrointestinal tract by bacteria Lactobacillus reuteri, in animals and found in foods of animal origin; Therefore, vegetarians are at increased risk for B12 deficiency. ${ }^{21,22}$

Vitamin B12 deficiency may cause pernicious anaemia and can lead to megaloblastic anaemia with demyelination of the central nervous system if not treated early. Due to high intake of folic acid the vitamin B12 status is masked in vegetarians and the deficiency of vitamin B12 may be difficult in diagnosis. It has been found that purported plant-based sources (tempeh, algae extracts, and sea vegetables) contain more inactive corrinoids than true vitamin B12 and thus are not reliable sources of B12. Vitamin B12 deficiency affects a person's mental and physical health and it can cause severe, irreversible neurologic damage, disorientation, nerve damage, insomnia and many other diseases. Vegetarian diet should be supplemented with fortified products (fortified yeast extract, fortified soya products, and breakfast cereals), the risk of vitamin B12 deficiency in vegans is decreased by adopting vitamin B12 sources in their diet.23-25

\section{Zinc}

Vegetarians are also seen at risk of zinc deficiency. Zinc deficiency has pronounced effect on the bone marrow which reduces the precursors of immune cells. ${ }^{26}$ While vegans have lower zinc intakes than omnivores, they do not vary in functional immunocompetence from non-vegetarians as measured by the natural cytotoxic activity of the killer cells. ${ }^{27}$ It appears that there may be facilitators of zinc absorption and 
compensatory mechanisms to help vegetarians adapt to a lower intake of zinc. ${ }^{28}$

\section{Iron}

Iron is found abundant in plants including kidney beans, soybeans, spinach, raisins, black beans, tomato juice, oatmeal, cashews, cabbage, but plant based diet containing iron has lower bioavailability than meat iron. ${ }^{29}$ Iron stores can be lower for those adopting a plant-based diet and eating little to no animal products. The American Dietetic Association, however, notes that anaemia with iron deficiency is uncommon even in individuals who adopt a plant-based $\operatorname{diet}^{30} \mathrm{Fe}$ intake in vegetarians and vegans is recommended to be higher, because plant sources of $\mathrm{Fe}$ (non-haem) are less bioavailable. In addition, in a plant-based diet, phytate, soya protein, and polyphenols/tannin can further inhibit $\mathrm{Fe}$ absorption. Ascorbic acid, retinol, alcohol, and carotenes can improve nonhaem Fe absorption. ${ }^{31}$

\section{Omega-3 Fatty Acids}

Essential fatty acids are fatty acids that humans need to consume for good health, as they are not synthesized by our bodies. Just two such essential fatty acids are known: linoleic acid (the fatty acid omega - 6) and alpha-linolenic acid (the fatty acid omega - 3). Only conditionally, three other fatty acids are essential: palmitoleic acid (a monounsaturated fatty acid), lauric acid (a saturated fatty acid), and gamma-linolenic acid (an omega - 6 fatty acid). Deficiencies in essential fatty acids can appear as an abnormality of the skin, hair and nail. ${ }^{32}$

The omega - 3 fats ( $\mathrm{n}-3$ fats) are the fatty acids that vegans are most likely to be deficient. Consumption of alpha-linolenic acid, a plant form of omega - 3 fats, are also small in vegans. Adequate consumption of $\mathrm{n}-3$ fats is associated with reduced heart disease and stroke incidence. It is worth highlighting foods that are strong sources of $\mathrm{n}-3$ fats which include seeds from ground flax, flax oil, walnuts, and canola oil. ${ }^{33}$ Alphalinolenic acid derived from plants can be converted in vivo to EPA and DHA but the conversion rate is low. ${ }^{34}$ As a result, lower serum levels of eicosapentaenoic acid (EPA) and docosahexaenoic acid (DHA) have been recorded in vegetables ${ }^{35.36}$ as well as lower omega - 3 erythrocyte phospholipids in vegetarians. ${ }^{35,36}$ Omega - 3 fatty acid vegan sources include flaxseed and flaxseed oil, canola oil, olive oil, and vegan supplement DHA. ${ }^{30}$

\section{Vitamin D}

Vitamin D plays an important role in the transport of calcium in the bone needed for healthy bone formation. Vitamin D exists naturally in animal products such as liver, fatty ocean fish, and egg yolks, so vegan diets can lack it. If fortified food intake is insufficient, strictly vegan infants and children may need supplementation in the form of vitamin D2 (ergocalciferol - a non-animal product). Supplementation is also necessary for all infants who are breastfed and for infants and children who consume less than $500 \mathrm{~mL}$ of fortified milk every day. For light-skinned children, sunlight exposure to the face and hands for $20 \mathrm{~min}$ to $30 \mathrm{~min} 3$ days a week is considered appropriate. Pigmented skin and the use of sunscreen minimize the effect of sunlight on vitamin D synthesis in the skin. ${ }^{37-39}$ Vegetarians can add fortified soy milk, mushrooms, fortified cereals, fortified orange juice, soy chunks, fortified almond milk, fortified rice milk, and the best thing to do is get out into the sun.

\section{Calcium}

Persons that do not consume plants that contain high levels of calcium can be at risk for mineralization and fractures of weakened bones. Research has however shown that the risk of fracture was high for vegetarians as well as non-vegetarians. Adequate calcium intake is the secret to bone health and seems to be independent of dietary preferences. ${ }^{40}$ Breast milk calcium intake is uninfluenced by a vegan diet in the mother. Nevertheless, maintaining sufficient intake of calcium-fortified foods such as fortified soy products, cereals, juices, mustard, turnip, greens and leafy vegetables is necessary after weaning. Highly bioavailable calcium for the older child is provided by low-oxalate greens (bok choy, Chinese cabbage, kale, collards etc). Spinach and some other plants contain calcium, which is bound to oxalate while abundant and is therefore poorly absorbed. ${ }^{41}$

\section{Protein}

Vegetarian diets can have less protein than a non-vegetarian diet; protein requirements may still be met. Many plant foods contain other proteins with the best sources being legumes, soy foods (including soy milk, soy yogurt, tofu, and tempeh), Quorn (mycoprotein), nuts, and seeds. Grains and vegetables produce protein too but in smaller amounts. Although the lower protein intake and protein consistency in a vegetarian diet is often thought to be a problem, there is growing evidence that eating protein from plant sources rather than animals could actually be one of the reasons why vegetarians typically have a lower risk of overweight, obesity and chronic diseases. Most plant protein sources are lower in cholesterol-free saturated fat and haem iron, and higher in fiber are good sources of antioxidants and phytochemicals, both of which may lead to reduced disease risk. ${ }^{42}$

\section{DISCUSSION}

Diet plays a significant role in people's overall health and health is considered as a primary concern for every individual. Knowing how poor dietary decisions can cause such issues can offer useful insight into common disorders, such as obesity, assessed by body mass index (BMI), diabetes, hypertension, and cholesterol-induced heart diseases. Those growing ailments can be connected by diet together. Bad food choices, for example, causes higher BMIs that can affect the sugar levels and eventually cause type 2 diabetes mellitus. High fat diet leads to heart disease and hypertension. Vegetarian diets have lower saturated fat and cholesterol and have higher amount of dietary fibre, magnesium, potassium, folate, carotenoids, flavonoids, vitamin $\mathrm{C}$ and other phytochemicals with many health benefits including lower blood glucose, cholesterol, blood-pressure and heart disease. Bone mineral density (BMD) and the possibility of bone fracture may be a problem when calcium and vitamin D are inadequately consumed. Eating calcium and vitamin D-fortified foods daily gives strength to bones. Vitamin B-12 deficiency is a potential issue for vegans; so the use of foods or supplements fortified with vitamin B-12 is important. To boost vegans, omega-3 fatty acid status, foods high in alpha lipoic acid (ALA), foods fortified with DHA, or supplements with DHA should be consumed daily. Vegans normally get sufficient intake of iron and don't 
suffer from anaemia more often than others. Vegan people will usually prevent health issues by making acceptable food choices. These tend to have at least as good health status as other vegetarians, such as lacto-ovo vegetarians. A vegetarian diet for all facets of life is not a quick-fix treatment nor should it be taken lightly. A vegetarian diet should be viewed as a way of influencing life, and a self-representation. There are many benefits obtained by eating a vegetarian diet, but when an individual wants to do on their own personal grounds, it will make the biggest difference.

\section{CONCLUSIONS}

The present review article concludes that the adoption of plant-based diets provides various benefits against a multitude of diseases without harmful side effects. A wellplanned plant-based diet is an easy and cost-effective method that can be used either when the disease is already present, or in combination with traditional treatment.

Financial or other competing interests: None.

Disclosure forms provided by the authors are available with the full text of this article at jemds.com.

\section{REFERENCES}

[1] Mitek M, Anyzewska A, Wawrzyniak A. Estimated dietary intakes of nitrates in vegetarians compared to a traditional diet in Poland and acceptable daily intakes: is there a risk? Roczniki Państwowego Zakładu Higieny 2013;64(2):105-9.

[2] Key TJ, Davey GK, Appleby PN. Health benefits of a vegetarian diet. Proceedings of the Nutrition Society 1999;58(2):271-5.

[3] Campbell TC, Campbell TM. The most comprehensive study of nutrition ever conducted and the startling implications for diet, weight loss and long-term health: the China study. Dallas, Texas: Benbella Books Publishing Company, 2006.

[4] Singh NP, Sabaté J, Fraser EG. Does low meat consumption increase life expectancy in humans? Am J Clin Nutr 2003;78(Suppl 3):526S-32S.

[5] Chauveau P, Combe C, Fouque D, et al. Vegetarianism: advantages and drawbacks in patients with chronic kidney diseases. Journal of Renal Nutrition 2013;23(6):399-405.

[6] Tonstad S, Butler T, Yan R, et al. Type of vegetarian diet, body weight and prevalence of type 2 diabetes. Diabetes Care 2009;32(5):791-6.

[7] Li D. Chemistry behind vegetarianism. Journal of Agricultural and Food Chemistry 2011;59(3):777-84

[8] Johnston CS. Vegetarian diet and possible mechanisms for impact on mood. In: Vegetarian and Plant-Based Diets in Health and Disease Prevention. Elsevier Inc., 2017: p. 493509.

[9] Wolters M. The significance of diet and associated factors in psoriasis. Der Hautarzt; Zeitschrift fur Dermatologie, Venerologie, und verwandte Gebiete 2006;57(11):9991004.
[10] Michaelsson G, Gerden B, Hagforsen E, et al. Psoriasis patients with antibodies to gliadin can be improved by a gluten-free diet. Br J Dermatol 2000;142(1):44-51.

[11] Michaelsson G, Kristjansson G, Lundin PI, et al. Palmoplantar pustulosis and gluten sensitivity: a study of serum antibodies against gliadin and tissue transglutaminase, the duodenal mucosa and effects of gluten-free diet. Br J Dermatol 2007;156(4):659-66.

[12] Takeshita J, Grewal S, Langan SM, et al. Psoriasis and comorbid diseases: implications for management. J Am Acad Dermatol 2017;76(3):393-403.

[13] Takeshita J, Grewal S, Langan SM, et al. Psoriasis and comorbid diseases: epidemiology. J Am Acad Dermatol 2017;76(3):377-90.

[14] Murray ML, Bergstresser PR, Adams-Huet B, et al. Relationship of psoriasis severity to obesity using samegender siblings as controls for obesity. Clin Exp Dermatol 2009;34(2):140-4.

[15] Butola LK, Meshram A, Dhok A, et al. Urticaria due to adverse drug reaction in diabetes--a case presentation. Journal of Evolution of Medical and Dental Sciences 2020;9(37):2764-7.

[16] McMacken M, Shah S. A plant-based diet for the prevention and treatment of type 2 diabetes. Journal of Geriatric Cardiology 2017;14(5):342-54.

[17] Clarys P, Deliens T, Huybrechts I, et al. Comparison of nutritional quality of the vegan, vegetarian, semivegetarian, pescovegetarian and omnivorous diet. Nutrients 2014;6(3):1318-32.

[18] Kahleova H, Levin S, Barnard ND. Vegetarian dietary patterns and cardiovascular disease. Progress in Cardiovascular Diseases 2018;61(1):54-61.

[19] Ferdowsian HR, Barnard ND. Effects of plant-based diets on plasma lipids. The American Journal of Cardiology 2009;104(7):947-56.

[20] Madigan M, Karhu E. The role of plant-based nutrition in cancer prevention. J Unexplored Med Data 2018;3:9.

[21] Madhura TK, Kanyal L, Mujawar A. Effect of glycemic control on vitamin b12 status in type 2 diabetes mellitus. Indian Journal of Basic and Applied Medical Research 2019;9(1):7-17.

[22] Kanyal L, Madhura TK. Status of vitamin b12 in type 2 diabetes mellitus patients taking metformin based oral hypoglycemic agent - a cross-sectional study. Indian Journal of Basic and Applied Medical Research 2019;9(1):18-26.

[23] Stabler SP, Allen RH. Vitamin B12 deficiency as a worldwide problem. Annu Rev Nutr 2004;24:299-326.

[24] Butola LK, Kute PK, Anjankar A, et al. Vitamin B12--Do you know everything? Journal of Evolution of Medical and Dental Sciences 2020;9(42):3139-46.

[25] Yajnik CS, Deshpande SS, Lubree HG, et al. Vitamin B12 deficiency and hyperhomocysteinemia in rural and urban Indians. J Assoc Physicians India 2006;54:775-82.

[26] Dhok A, Butola LK, Anjankar A, et al. Role of Vitamins and Minerals in improving immunity during Covid-19 pandemic--a review. Journal of Evolution of Medical and Dental Sciences 2020;9(32):2296-300.

[27] Haddad EH, Berk LS, Kettering JD, et al. Dietary intake and biochemical, hematologic and immune status of vegans compared with nonvegetarians. Am J Clin Nutr 1999;70(Suppl 3):586S-93S. 
[28] Gibson RS. Content and bioavailability of trace elements in vegetarian diets. Am J Clin Nutr 1994;59(Suppl 5):1223S-32S.

[29] Waldmann A, Koschizke JW, Leitzmann C, et al. Dietary iron intake and iron status of German female vegans: results of the German vegan study. Ann Nutr Metab 2004;48(2):103-8.

[30] Craig WJ, Mangels AR. American Dietetic Association. Position of the American Dietetic Association: vegetarian diets. J Am Diet Assoc 2009;109(7):1266-82.

[31] Hunt JR. Bioavailability of iron, zinc and other trace minerals from vegetarian diets. Am J Clin Nutr 2003;78(Suppl 3):633S-9S.

[32] Rosell MS, Lloyd-Wright Z, Appleby PN, et al. Long-chain $\mathrm{n}-3$ polyunsaturated fatty acids in plasma in British meateating, vegetarian and vegan men. Am J Clin Nutr 2005;82(2):327-34.

[33] Davis BC, Kris-Etherton PM. Achieving optimal essential fatty acid status in vegetarians: current knowledge and practical implications. Am J Clin Nutr 2003;78(Suppl 3):640S-6S.

[34] Pawlosky RJ, Hibbeln JR, Novotny JA, et al. Physiological compartmental analysis of a-linolenic acid metabolism in adult humans. J Lipid Res 2001;42(8):1257-65.
[35] Craig WJ. Health effects of vegan diets. Am J Clin Nutr 2009;89(5):1627S-33S.

[36] Sanders TAB. DHA status of vegetarians. Prostaglandins Leukot Essent Fatty Acids 2009;81(2-3):137-41.

[37] Lata KB, Neelam G. To study the association of Vitamin D, Calcium and Phosphorus in type 2 diabetes mellitus patients. Int J Recent Sci Res 2020;11(10):39749-52.

[38] Neelam G, Lata KB. The cross- sectional population-based study conducted to elucidate the prevalence of Vitamin D deficiency, bone turnover Marker's variation among adolcents of Haryana. Int J Recent Sci Res 2020;11(8):39631-4.

[39] Amit M, Canadian Paediatric Society, Community Paediatrics Committee. Vegetarian diets in children and adolescents. Paediatrics \& Child Health 2010;15(5):30314.

[40] Appleby P, Roddam A, Allen N, et al. Comparative fracture risk in vegetarians and non-vegetarians in EPIC-Oxford. Eur J Clin Nutr 2007;61(12):1400-6.

[41] Weaver CM, Plawecki KL. Dietary calcium: adequacy of a vegetarian diet. Am J Clin Nutr 1994;59(Suppl 5):1238S41S.

[42] Marsh KA, Munn EA, Baines SK. Protein and vegetarian diets. Med J Aust 2013;199(Suppl 4):S7-10. 